\title{
Frontières
}

\section{Mourir deux fois}

Le devenir du corps des soldats sur les champs de bataille pendant la Première Guerre mondiale

\section{Serenella Nonnis Vigilante}

Volume 19, numéro 1, automne 2006

Enjeux politiques et mort

URI : https://id.erudit.org/iderudit/016641ar

DOI : https://doi.org/10.7202/016641ar

Aller au sommaire du numéro

\section{Éditeur(s)}

Université du Québec à Montréal

ISSN

1180-3479 (imprimé)

1916-0976 (numérique)

Découvrir la revue

Citer cet article

Vigilante, S. N. (2006). Mourir deux fois : le devenir du corps des soldats sur les champs de bataille pendant la Première Guerre mondiale. Frontières, 19(1),

80-84. https://doi.org/10.7202/016641ar d'utilisation que vous pouvez consulter en ligne.

https://apropos.erudit.org/fr/usagers/politique-dutilisation/ 


\section{MOURIR DEUX FOIS Le devenir du corps des soldats sur les champs de bataille pendant la Première Guerre mondiale}

\author{
Serenella Nonnis Vigilante, \\ maître de conférences, Université Paris XIII.
}

Pendant la Première Guerre mondiale, l'échafaudage législatif qui gère les sépultures, bâti à partir du décret de Prairial an XII du Code napoléonien, s'effondre souvent dans les pratiques. Il s'effondre alors que, dans la société civile, se célèbre le triomphe de la mort bourgeoise dont les éléments fondateurs sont l'hygiène, la piété et la mémoire ${ }^{1}$. L'historiographie contemporaine montre que, sur les champs de bataille, les sépultures demeurent souvent rudimentaires, confiées au hasard, loin du respect des normes d'hygiène, ainsi que du culte des morts ${ }^{2}$.

\section{L'EFFONDREMENT DES RÈGLES}

Enchevêtrés avec les charognes des chevaux, jetés dans des fosses communes et rapidement recouverts de chaux vive et de terre, de la même manière que les animaux, ou bien ensevelis sur les bords des routes, à la lisière ou au milieu des bois, dans les champs, dans les jardins, parmi les ruines, ou encore livrés aux corbeaux et abandonnés à la décomposition, les corps morts des soldats n'existent plus.

Sur le champ de bataille, le soldat qui ne reçoit pas la sépulture codifiée par le législateur meurt deux fois. Il meurt deux fois, car son cadavre perd son statut juridique, c'est-à-dire la protection de la loi, et lui, le soldat en tant qu'individu, perd son caractère de défunt ayant droit à la mémoire civique individuelle. Il s'agit des droits que les morts ont acquis depuis le décret de Prairial an XII évoqué. Ce décret, constamment rappelé dans les dispositifs mortuaires conçus par les autorités militaires durant la Première Guerre mondiale, est le fruit d'une convergence heureuse entre les exigences des élites médicales positivistes et hygiénistes, des élites politiques et de l'Église catholique. À partir de là, au long du XIX ${ }^{\mathrm{e}}$ siècle, se construit progressivement la politique mortuaire moderne tendant à la médicalisation et à la laïcisation de la mort.

En Europe, les principes posés en 1804 par le décret napoléonien avaient marqué un tournant dans les dispositifs mortuaires, apportant une réponse à deux grands problèmes : la séparation définitive des vivants et des morts et le choix, pour le cadavre, de l'emplacement le plus conforme aux règles de l'hygiène collective et au respect de la piété du défunt. L'arsenal des lois et des règlements qui régissent maintenant les pratiques mortuaires en France révèle la volonté des élites de mettre en œuvre un projet pédagogique extrêmement cohérent. Ce projet réglemente toutes les phases après 


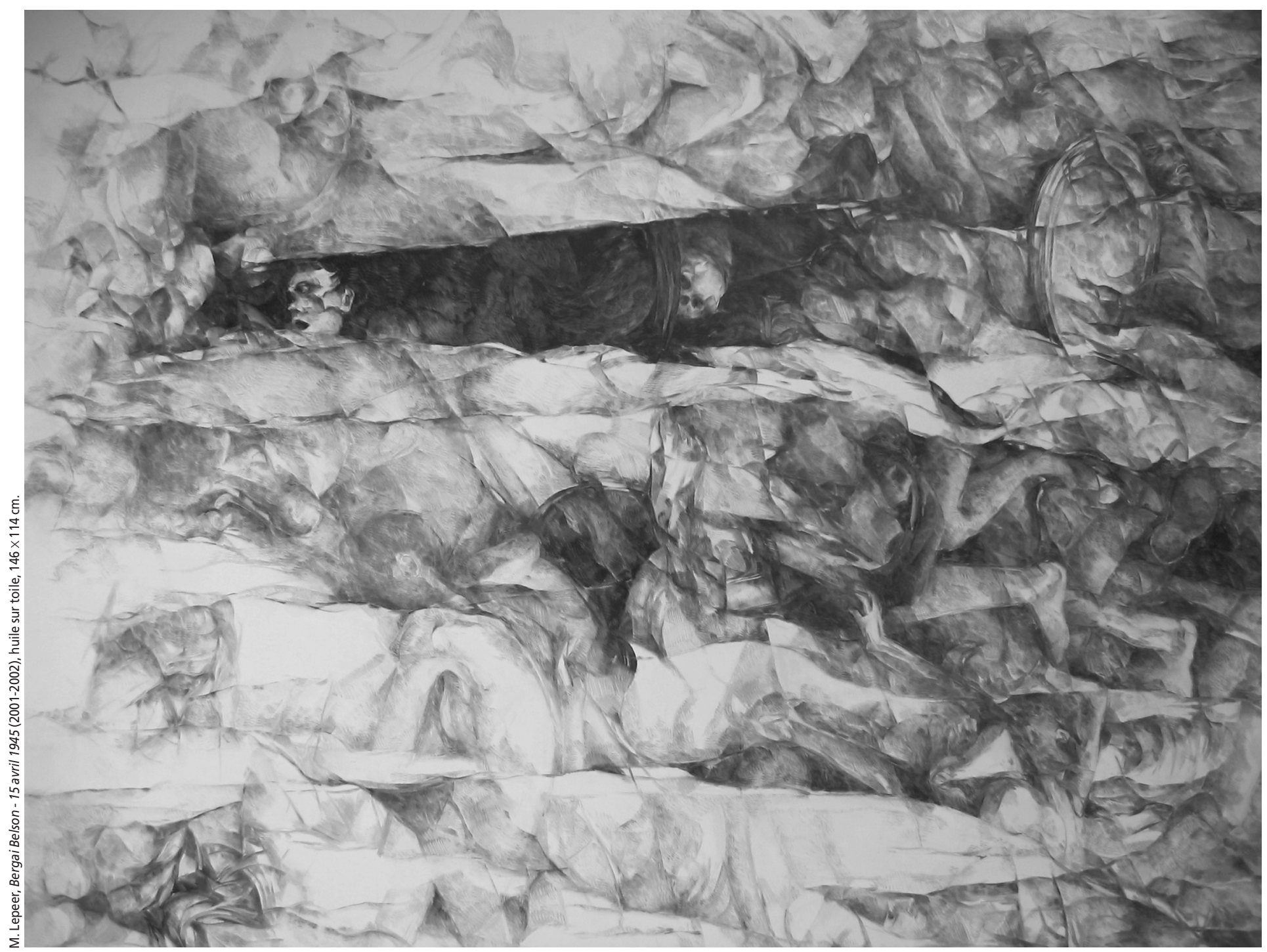

la mort: ce qu'il faut faire au domicile du défunt, au cours de son transfert à l'église et au cimetière, au cours de l'enterrement lui-même. La mort doit être déclarée à la commune, le décès doit être certifié par un médecin. Le cadavre doit rester vingt-quatre heures chez lui, placé dans un endroit à l'écart du reste de la maison. On ne doit pas le toucher: seul un personnel spécialisé envoyé par l'administration communale peut le bouger. Ce personnel est aussi autorisé à le mettre en bière, à le transporter et à l'enterrer.

Ainsi, en l'espace d'environ un siècle et demi, entre accélérations et résistances, se réalise la mise en place d'une gestion publique et laïque de la mort. Le corps mort est définitivement soustrait à l'Église et sorti de l'anonymat dans lequel l'avait confiné la pratique séculaire des fosses collectives, et alors qu'une nouvelle célébration de la mémoire voit le jour. À partir de là les demandes de concession d'inhumation perpétuelle se multiplient, ainsi que pour la construction de modestes columbariums ou de tombeaux dotés d'épitaphes, de statues, de bustes et de colonnes, exaltant les vertus civiques et familiales du défunt. La bourgeoisie du XIX $^{\mathrm{e}}$ siècle célèbre ainsi la cohésion sociale à travers l'affirmation des valeurs de la famille, du travail, de la religion et de la patrie (Nonnis-Vigilante, 2005, p. 129-164).

Mais, dès les années 1870, à l'issue de la guerre franco-allemande et encore pendant la Première Guerre mondiale, les élites politiques, scientifiques et militaires comprennent que les pratiques mortuaires mises en œuvre sur les champs de bataille sont incompatibles avec celles que la société tout entière a désormais intériorisées. Nous avons retenu ici l'angle d'observation des médecins et des scientifiques (notamment les chimistes), directement impliqués dans la tâche difficile de libérer les terrains des corps des soldats morts, abandonnés ou ensevelis hâtivement par leur camarades après la bataille. Que faire de ces corps? Comment s'en débarrasser d'une façon qui ne nuise pas à la santé des vivants? Comment concilier la dignité de ces cadavres avec le respect des défunts? Comment protéger la sensibilité des familles qui ignorent le sort réservé au corps de leur proches? Telles sont les questions que ces élites se posent, sans pour autant trouver de véritables solutions.

Leurs discours expriment deux attitudes opposées et complémentaires. L'attitude, qu'on pourrait qualifier d'hygiéniste à tout prix, cherche à protéger la santé des vivants menacée par les infections provenant de la décomposition des cadavres, au détriment du respect du défunt. Cet esprit hygiéniste justifie la pratique des sépultures rapides, de l'enfouissement des corps dans des fosses communes et de leur incinération, sans autre forme de procès, en méconnaissant les risques, tout à fait réels, de morts apparentes et de sépultures prématurées. La seconde attitude, qui s'inscrit dans le registre du culte des morts et de la sensibilité collective, déplore la perte d'identité des soldats, ainsi que de la dignité des défunts, et pense aux familles cruellement privées 
des corps de leurs proches et auxquels elles souhaiteraient, au moins, donner une sépulture digne.

\section{POUR LA SANTÉ DES VIVANTS: ENSEVELIR, COUVRIR DE CHAUX VIVE, BRÛLER}

En 1917, la rédaction de la Revue d'Hygiène et de Police sanitaire estime que les circonstances de guerre donnent «un intérêt tout particulier» à la reproduction du mémoire du chimiste Créteur, présenté par l'auteur en 1876, au premier Congrès international d'Hygiène et de Démographie de Bruxelles. Créteur est chargé par le gouvernement belge de l'assainissement de divers terrains où des centaines de corps gisent abandonnés sept mois après la bataille de Sedan.

La réactualisation de son expérience, à presque un demi-siècle de sa mise en œuvre, semble particulièrement révélatrice de l'absence de nouvelles normes de sépultures à laquelle se trouve alors confrontée l'Armée. En 1871, comme dans les années 19151918, les urgences dictées par les normes d'hygiène et de santé publique demeurent identiques: défendre les populations des infections engendrées par la proximité des cadavres humains et des charognes de chevaux, les libérer des miasmes insupportables provenant de la décomposition de la chair, assainir les terrains et les rendre le plus rapidement possible à l'agriculture.

Lorsque Créteur arriva sur les lieux d'affrontement dans la région de Sedan, la variole et le typhus y sévissaient. Le spectacle qui s'offrait à ses yeux était spectral. Les corbeaux et les oiseaux lui indiquaient où les soldats gisaient. La plupart des milliers de cadavres étaient désormais en décomposition, à demi-enfouis, abandonnés en plein champ ou bien découverts par le travail même de la décomposition; d'autres avaient les bras et les jambes sortis des fosses, à moitié dévorés par les chiens et les oiseaux.

"On se figure difficilement ce que c'est qu'un champ de bataille, écrit Créteur, on pense parfois que l'action se concentre sur une plaine de peu d'étendue ou aux environs d'une place de guerre. C'est une erreur. Pour faire mourir des centaines de milliers

d'hommes, il faut de l'espace et cet espace embrasse souvent une étendue de 20 à 25 lieues, sur laquelle se livre une succession de combats. »

Une chose lui apparaît évidente: étant donné la quantité de cadavres et l'étendue de terrain qu'il aurait fallu pour creuser les tombes, l'enterrement des corps un à un était impossible. Comme, selon ses calculs, il fallait «réduire cent cadavres en moins d'une heure », il n'avait d'autre choix que de procéder à l'incinération des corps à l'aide du goudron de houille. Ainsi, fait-il enlever la terre des tumulus jusqu'à la première couche des cadavres pour la faire recouvrir d'une couche de chlorure de chaux, afin de neutraliser la mauvaise odeur. Puis, audessus de la couche de chlorure de chaux, il fait couler du goudron et allume ensuite le tout à l'aide d'huile de pétrole. Le pétrole enflammé s'étend alors sur toute la couche de goudron, qui entre immédiatement en ignition, attaquant les chairs et faisant fondre les graisses; les corps gras, en se mêlant au goudron, ont élevé la température propre à réduire rapidement les cadavres en cendres. Une fois le travail terminé, dans le fond des fosses ne restaient que les os les plus volumineux, recouverts d'une couche de brai sec.

Pour faire disparaître tous les corps, Créteur travaille pendant quatre mois avec l'aide de 150 ouvriers : "Mon procédé était simple, mais énergique, précise le chimiste, et je crois qu'aucun appareil ne produirait le même résultat ${ }^{3}$.» Par cette réflexion, le chimiste fait allusion à la crémation des corps à l'aide d'un four crématoire, une pratique de sépulture qui, depuis quelque années, était débattue dans la société civile en France comme en Allemagne, en Belgique et en Italie, où elle alimentait des polémiques dans les milieux scientifiques, politiques et religieux.

Pendant ces décennies, les promoteurs de la crémation sont des scientifiques appartenant au groupe le plus radical du mouvement hygiéniste. Il s'agit surtout d'hommes appartenant à la franc-maçonnerie (dont le dessein est la laïcisation de la société), qui envoient ainsi un message provocateur et en même temps symbolique aux milieux conservateurs catholiques de la société européenne: l'hygiène concerne tous les moments de la vie du citoyen, y compris la mort. Le citoyen nouveau, aspiration d'une société tournée vers plus de démocratie et plus de laïcité, doit être vacciné, désinfecté, soigné, lavé et, pourquoi pas, incinéré, au nom de l'intérêt général de la collectivité.

Du débat crématiste du XIX ${ }^{\mathrm{e}}$ siècle émergent trois principes: la pratique de la réduction du corps en cendres préserve de l'horreur de la putréfaction, rend la terre aux vivants et libère les villes des miasmes des cimetières (que certains hygiénistes considèrent encore comme des lieux peu sûrs du point de vue infectieux). Le four crématoire permet de diffuser l'image d'une science qui donne à la société les instruments de son propre progrès.

$\mathrm{Si}$, dans les années 1880 et 1890 , les gouvernements des pays d'Europe occidentale codifient par les lois de santé publique la liberté des individus de choisir la desti- nation de leur propre corps après la mort (autrement dit de choisir entre l'inhumation et la crémation), les hiérarchies ecclésiastiques ne suivent pas les crématistes. L'Église n'ignore pas que l'enjeu va bien au-delà de la simple question d'hygiène, puisque la pratique de la crémation touche directement à la religiosité populaire. Sa réaction ne laisse aucune place à la réussite du projet: l'excommunication - prononcée par le pape Léon XIII en 1886 -, de tous ceux qui choisissent de se faire incinérer bloque toute velléité de changement: la pratique demeure longtemps largement minoritaire parmi les populations, tandis que le débat continue parmi les élites, tout en ayant perdu l'esprit combattant de la première heure (Nonnis-Vigilante, 1998, p. 140-176 et 2005, p. 79-90)

La Première Guerre mondiale donne l'occasion aux militants des sociétés de crémation de proposer encore une fois la réduction rapide des cadavres, toujours sous le prétexte du respect de l'hygiène des sépultures. En 1915, le docteur Georges Salomon, secrétaire général de la Société de crémation pour la propagation de l'incinération de Paris, remarque que les normes promulguées pour la salubrité de l'inhumation dans les cimetières ne sont pas applicables sur les champs de bataille. Ses arguments sont incontestables: au lendemain des grands combats, on n'a ni le temps, ni les moyens de se livrer à une étude géologique du sol, des eaux souterraines, de l'altitude, des vents dominants, de l'éloignement des habitations, des sources et des puits; on n'a ni assez de temps, ni assez de bras pour procéder à l'ensevelissement des cadavres, creuser des fosses individuelles ou même des tranchées aux dimensions réglementaires. Et si, dans les cimetières publics, les corps sont apportés par les fossoyeurs communaux aux bords mêmes de la fosse, sur les champs de bataille, les brancardiers militaires chargés des inhumations doivent les chercher de tous côtés, au travers des prés et des bois, jusqu'au fond des marais. En effet, lors des combats de la Marne et de l'Ourcq, le nombre de morts dépassa tellement les prévisions qu'il a fallu adjoindre, comme fossoyeurs, aux brancardiers, des hommes de troupe complètement inexpérimentés. Ainsi, un mois après la bataille, le sol était-il encore couvert de corps. Une bonne solution aurait pu être celle de la destruction, "aussitôt sur place, soit par la chaux anhydre, soit moins par le feu, les corps relevés, dès l'issue du combat, ou soit d'en inhumer ensuite les restes après stérilisation suffisante» (Salomon, 1915, p. 548-549).

La grave impasse dans laquelle se trouve l'Armée alimente, chez les crématistes, l'espoir que soient utilisés des wagons créma- 
toires. Ces wagons auraient pu transporter les fours d'un lieu de combat à l'autre, de manière à incinérer les corps au fur et à mesure que les soldats tombaient au combat. Cependant, les discours tout à fait rationnels des crématistes se heurtent encore à une série d'obstacles. En premier lieu, des obstacles de nature logistique et économique: nombre de wagons auraient dû suivre les combattants (au risque de décourager les jeunes soldats) et occuper des kilomètres de chemin de fer, un engagement des municipalités pour fournir des ambulances; un nombre considérable de corps auraient dû être réunis en des points déterminés et il aurait fallu accumuler une énorme quantité de combustible. Le docteur Fiessinger, médecin légal de l'Armée, visiblement hostile à cette pratique mortuaire précise ainsi: «Peu de personnes savent combien l'incinération d'un corps humain, même pratiquée dans les fours crématoires les mieux installés, est toujours longue et compliquée, il faut une heure et demie au moins pour réduire un cadavre en cendre blanche, l'opération est de pratique irréalisable. (Fiessinger, 1915, p. 381.)

À ces objections convaincantes s'ajoutent des considérations morales: l'organisation anticipée de la réduction des corps avait quelque chose de sinistre: «Écartons tout d'abord les arguments sentimentaux et religieux, écrit en 1915 le médecin militaire Viry, encore devrait-on peut-être tenir compte de l'opinion des intéressés, en espèce les soldats eux-mêmes. » (Viry, 1915, p. 458.)

Mais si la crémation des corps à l'aide d'un four est refusée par l'Armée, celle de l'incinération des corps par le bûcher avec adjonction de pétrole ou de toute autre substance capable d'activer la combustion et la destruction par la chaux vive, demeure envisagée. Les hiérarchies militaires auraient voulu partager cette lourde responsabilité avec le législateur, mais en fin de compte elles se sont retrouvées seules. En 1917, le Parlement méconnaissait la gravité exceptionnelle du problème dont il avait été saisi.

\section{LA PERTE DE L'INDIVIDU, L'ABSENCE DU DÉFUNT}

En 1917, le médecin Bosredon, aidemajor de l'Armée, dans un article paru dans la revue Archives de Médecine et de Pharmacie militaire, précise que le seul document militaire traitant des inhumations ne prévoit que des mesures d'hygiène à prendre après le combat. Avant la guerre mondiale, on croyait pouvoir procéder aux inhumations sous la protection des règlements internationaux de la Convention de Genève. Tout était «soigneusement » codifié pour élire l'emplacement des corps morts

des soldats, ainsi que pour transmettre leur mémoire: le choix d'un terrain convenable, l'inhumation dans des fosses de 10 mètres de long sur 2 de large et 2 de profondeur, où l'on superposerait les cadavres sur trois ou quatre rangs de manière qu'ils se décomposent rapidement sans porter atteinte à la santé des vivants; un "poteau» ou un autre signe apparent quelconque, des plantes avides d'azote, voilà ce qui avait été prévu pour « repérer et orner ces fosses communes où devaient dormir les héros ». Mais dès la première phase de la guerre, rien ne se déroula comme on l'avait imaginé. Écoutons le récit de Bosredon:

On fait comme l'on peut pour le mieux tout est permis, tout est excusable. On se débarrasse des cadavres, lorsqu'ils deviennent trop gênants et qu'ils incommodent les combattants. On profite d'un moment d'accalmie, des ombres de la nuit, pour les traîner jusqu'à un trou d'obus, jusqu'à une tranchée abandonnée, où dans un abri aboulé, où on les enterre sommairement. On en remplit les caves et les casemates que l'on fait murer ensuite. Ce ne sont plus des inhumations, c'est bien de l'enfouissement! (Bosredon, 1917, p. 568.)

\title{
LE CITOYEN NOUVEAU, ASPIRATION D'UNE SOCIÉTÉ TOURNÉE
} VERS PLUS DE DÉMOCRATIE ET PLUS DE LAÏCITÉ, DOIT ÊTRE VACCINÉ,

\author{
DÉSINFECTÉ, SOIGNÉ, LAVÉ ET, POURQUOI PAS, INCINÉRÉ, \\ AU NOM DE L'INTÉRÊT GÉNÉRAL DE LA COLLECTIVITÉ
}

Ce mode de sépulture, qui ne tient pas compte des droits juridiques du cadavre, efface aussi l'identité de l'individu et empêche la construction du défunt. Aucune mémoire visible et tangible (une tombe) ne peut se construire pour l'individu disparu. En premier lieu, à cause des conditions déplorables dans lesquelles se font les identifications qui sont «rudimentaires, défectueuses, insuffisantes» (Chavigny, 1917, p. 33). Des officiers, des soldats, des gendarmes, des gens bien intentionnés parcourent les champs de bataille, fouillent les morts pour leur enlever leur plaque d'identité, conformément aux règlements. Les équipes chargées des inhumations se trouvent en présence de cadavres d'inconnus. Toutefois, elles n'ont pas à s'en préoccuper puisque tous les corps doivent être ensevelis dans une tombe anonyme.

En 1915, consciente du drame qui se produit et pour répondre à «l'oubli» du règlement, l'Armée promulgue une série de normes visant à rendre possible l'identification des militaires décédés. Les soldats auraient dû porter sur eux deux plaques, une aurait été prélevée au moment de l'inhumation et l'autre aurait été laissée sur le cadavre afin de permettre la reconnaissance de la personne au moment de l'exhumation. L'année suivante, un carnet personnel se rajoute aux plaques; toutefois aucune mesure n'apportera de solution définitive, car le premier souci est de repérer le lieu où se trouveraient les tombes.

En 1917, le règlement des sépultures n'a toujours pas prévu de moyens de repérage. On laisse la tâche à l'initiative de chacun, reconnaît Bosredon:

Une croix en bois, plus au moins façonnée, est le mode de repérage le plus fréquemment employé, on y trace au crayon, à l'encre, on y grave au couteau, au fer rouge, suivant les moyens dont on dispose, les inscriptions nécessaires. Le grand nombre des militaires portés disparus et de tombes anonymes est la preuve indiscutable de l'insuffisance des moyens d'identification (Bosredon, 1917, p. 60.)

On comprend alors qu'en 1918, toujours dans les pages du Journal de médecine de Bordeaux, on s'interroge sur les conséquen- ces morales que ces sépultures ont pour la famille. Ainsi, à mesure que les années de guerre passent, les médecins militaires se sentent de plus en plus moralement coresponsables de cette pratique de sépulture, même s'ils n'y sont pas directement impliqués. Ces médecins ne peuvent s'empêcher de penser aux familles qui, de cette manière, endurent deux fois la mort de leurs proches. C'est bien ce que pense la fille d'un général, venue en 1917 sur le champ de bataille de la Marne rechercher le corps de son frère. Elle dit «qu'il lui semblait le perdre une seconde fois en apprenant que jamais le corps mêlé à tant d'autres ne pourrait être retrouvé » (Chavigny, 1917, p. 39)

En 1918, le docteur Bosredon paraît assez exemplaire des sentiments contrastés qui traversent les hiérarchies de l'Armée:

Comment a-t-on pu méconnaître

à ce point le culte et la vénération que notre Nation a pour ses morts? Pouvait-on ignorer que les familles 
viendraient, un jour, réclamer le corps de leurs enfants pour le ramener au cimetière de leur village? On se rend compte que plus la guerre se prolonge, plus la paix se rapproche de la nécessité d'assurer aux soldats une sépulture digne d'eux. C'était une dette de reconnaissance, que les vivants doivent payer à ceux qui avaient donné leur vie à leur pays. Préparons-nous à l'acquitter. Au lendemain de la paix n'ajoutons pas cette préoccupation à tant d'autres qui nous assaillirons. Il est grand temps qu'on étudie et qu'on réglemente cette question; il faut envisager la constitution de saints lieux de pèlerinage où reposeront nos immortels héros. Les familles y viendront pleurer avec une vive douleur, que relèvera un légitime orgueil, leurs enfants tombés pour la patrie. (Bosredon, 1918, p. 61.)

Ainsi, avant que la guerre se termine, ces témoins privilégiés de la disparition des corps des combattants apportent leur contribution à l'affirmation dans la société civile de la rhétorique du mythe du soldat inconnu.

\section{Biliographie}

ARIÈS, Ph. (1975), Essai sur l'histoire de la mort en Occident du Moyen Âge à nos jours, Paris, Seuil.

AUDOIN-ROUZEAU, S. et A. BECKER (2000), 14-18 - Retrouver la guerre, Paris, Gallimard.

BARRIER, M. (1915), "La crémation des cadavres sur les champs de bataille», Revue d'Hygiène et de Police sanitaire, v. XXVII, p. 545-557.

BARRIER, M. (1915), "L'incinération en temps de guerre», Journal des praticiens, $\mathrm{n}^{\circ} 27$, p. $417-19$

BOSREDON, M. (1917), «Les inhumations aux armées. Ce qu'elles devaient être. Ce quelles sont. Ce qu'elles devraient être», Archives de Médecine et de Pharmacie militaire, $\mathrm{n}^{\circ} 3$, p. 561-581.

BOSREDON, L. (1918), "Les inhumations des militaires à Bordeaux ", Journal de médecine de Bordeaux, $\mathrm{n}^{\circ} 3$, p. 55-62

CAPDEVILA, L. et VOLDMAN, D. (2002), Nos morts. Les sociétés occidentales face aux tués de la guerre, Paris, Payot.

CHAVIGNY, D ${ }^{r}$ (1917), «De l'identification des individus particulièrement en temps de guerre ", Annales d'hygiène et de médecine légale, t. XXVIII, p. 32-40.

DOIZY, M. (1915), Les nécessités d'hygiène dans les lieux de combat et d'occupation », Revue scientifique, 32, p. 101-102.

FIESSINGER, C. (1915), "Médecine légale de l'Armée », Journal des praticiens, $\mathrm{n}^{\circ} 27$, p. 380-382

NONNIS VIGILANTE, S. $\left(1998 \mathrm{II}^{\circ}\right)$, I cittadini tra inumazione e cremazione (1998) dans A. Comba, E. Mana et S. Nonnis Vigilante, $L a$ morte laica. Storia della cremazione Torino (1880-1920), Torino, Paravia.
NONNIS VIGILANTE, S. (2004), «Pour une histoire de la crémation en Italie. XIX-XX siècles », "Études sur la mort», Revue de la Société de thanatologie, Le monde des funérailles, $\mathrm{n}^{\mathrm{o}} 125$.

NONNIS VIGILANTE, S. (2005), «Les intolérables des politiques mortuaires modernes. Le corps mort entre religion et hygiène, en France et en Italie - XVIII ${ }^{\mathrm{e}}-\mathrm{XXI}{ }^{\mathrm{e}}$ siècles ", dans D. Fassin et P. Bourdelais (dir.), Les constructions de l'intolérable. Études d'anthropologie et d'histoire sur les frontières de l'espace moral, Paris, La Découverte.

SALOMON, G. (1915), «Discours prononcé à l'Assemblée genérale de Société pour la propagation de l'incinération ", Revue d'Hygiène et de Police sanitaire, v. XXVII, 1915, p. 349-360.

VAN ROOY, M. (1877), «Questions des animaux blessés ou errants sur les champs de bataille », Congrès international d'Hygiène, de Sauvetage et d'Économie sociale, v. II, Paris, Germer Baillière, p. 330-336.

VOVELLE, M. (1974), Mourir autrefois, Paris, Gallimard-Julliard.

\section{Notes}

1. Nous renvoyons aux travaux pionniers de M. Vovelle, Mourir autrefois, Paris, GallimardJulliard, 1974, et de Ph. Ariès, Essai sur l'histoire de la mort en Occident du Moyen Âge à nos jours, Paris, Seuil, 1975.

2. Cf. S. Audoin-rouzeau et A. Becker, 14-18 - Retrouver la guerre, Paris, Gallimard, 2000 et $c f$. Luc Capdevila et Danièle Voldman, Nos morts. Les sociétés occidentales face aux tués de la guerre, Paris, Payot, 2002.

3. Le mémoire de Créteur «La pratique de la crémation des cadavres sur les champs de la bataille de Sedan en 1871 par M. Créteur » est publié dans la Revue d'Hygiène et de Police sanitaire, vol. XXVII, a.1915, p. 558-562. 